\title{
Prévalence du syndrome métabolique chez les sujets hypertendus adultes dans les formations sanitaires de Parakou (Bénin)
}

\author{
M. GOMINA ASSOUMANOU ${ }^{1 *}$, A. C. DOVONOU ${ }^{2}$, M. R. NGOME $^{1}$ et \\ S. A. AKPONA ${ }^{1}$ \\ ${ }^{1}$ UER de Biochimie et de Biologie Moléculaire, Faculté de Médecine, Université de Parakou, \\ BP : 123 Parakou (Bénin). \\ ${ }^{2}$ Service de Médecine Interne, Centre Hospitalier Départemental et Universitaire de Parakou, \\ BP : 02 Parakou (Bénin). \\ *Auteur correspondant, E-mail : elboutraguero@yahoo.fr, Tel : + 22995966940
}

\section{RESUME}

Le syndrome métabolique (SM) est un groupe d'anomalies qui confère un risque accru de développer les maladies cardiovasculaires et le diabète de type 2. L'objectif de cette étude était de déterminer la prévalence du SM chez les hypertendus adultes dans les formations sanitaires de Parakou selon les critères de la Fédération Internationale du Diabète (FID) et de la National Cholesterol Education Programme (NCEP) et d'évaluer la concordance entre ces deux critères. A travers une étude descriptive transversale avec recueil prospectif des données menée du 20 février au 30 juin 2010, 270 sujets hypertendus adultes (150 femmes, 120 hommes) ont été recrutés dans huit formations sanitaires de Parakou (Bénin). Chaque sujet de l'étude a bénéficié de mesures suivantes: poids, taille, pression artérielle, glycémie, cholestérolémie HDL et triglycéridémie. La prévalence du SM selon les critères de la FID était de 69,6\% contre 65,2\% selon ceux de la NCEP. Le taux de prévalence était significativement plus élevé chez les femmes que chez les hommes indépendamment des critères utilisés (les deux $\mathrm{p}<0,001$ ). La concordance entre les deux définitions était moyenne : 0,41 (intervalle de confiance à $95 \%$ : 0,30 - 0,53).

(c) 2012 International Formulae Group. All rights reserved.

Mots clés: hypertension artérielle, glycémie, triglycéridémie, FID, NCEP.

\section{INTRODUCTION}

Le syndrome métabolique (SM) est un groupe d'anomalies qui font courir un risque accru de développer les maladies cardiovasculaires et le diabète de type 2 (Grundy et al., 2004). Durant cette dernière décennie, cinq définitions ont été proposées par plusieurs groupes pour caractériser le SM (Kelliny et al., 2008). A l'exception de la définition de l'American Association of
Clinical Endocrinologists/American College of Endocrinology (AACE/ACE), toutes les autres définitions se basent sur l'obésité centrale, l'hypertension artérielle, la dyslipidémie, l'intolérance au glucose ou l'insulino-résistance. Toutefois, les seuils de définition des facteurs de risque varient suivant les définitions. La conséquence de l'hétérogénéité des composantes du SM est que, en considérant toutes ces définitions, il 
serait possible de diagnostiquer des groupes différents de patients au sein d'une population donnée. Ceci aurait des conséquences néfastes pour la définition des stratégies de prévention des affections liées à ce syndrome.

L'hypertension artérielle (HTA) est une composante importante du SM qui se rencontre avec une forte prévalence chez les obèses et les diabétiques de type 2. Elle est la principale cause de mortalité cardiovasculaire dans le monde et plus particulièrement des accidents vasculaires cérébraux (Kannel, 2000). La coexistence de l'HTA avec d'autres facteurs de risque métaboliques potentialise le risque de survenue de maladies cardiovasculaires (Kannel, 2000).

De grandes variations de la prévalence du SM ont été rapportées d'un pays à un autre, entre les sexes d'une part et les groupes ethniques d'autre part (Athyros et al., 2005 ; Ford, 2005 ; Pollex et al., 2006 ; Fezeu et al., 2007 ; Hydrie et al., 2009). Si les données sur la prévalence du SM chez les patients hypertendus sont rares de part le monde (Chih-Neng et al., 2005 ; Bulhões et Araújo, 2007 ; Akintunde et al., 2011), elles sont inexistantes au Bénin. De plus la performance comparée des différents critères de définition demeure inconnue.

L'objectif de notre travail était de déterminer la prévalence du SM chez les hypertendus adultes dans les formations sanitaires de Parakou (Bénin) selon les critères de la Fédération Internationale du Diabète (FID) et de la National Cholesterol Education Programme (NCEP) et d'évaluer la concordance entre ces deux critères.

\section{MATERIEL ET METHODES}

\section{Ethique}

Nous avons réalisé une étude transversale à visée descriptive avec recueil prospectif des données du 20 février au 30 juin 2010. Le protocole a été validé par le Comité National Provisoire d'Ethique de la Recherche en Santé du Bénin et les sujets de la présente étude ont tous donné leur consentement libre et éclairé.

\section{Population d'étude}

Notre échantillon était composé de patients hypertendus adultes consultant dans huit formations sanitaires de Parakou : Centre Hospitalier Départemental du Borgou, clinique "AS du CCEUR", clinique médicochirurgicale, clinique "Santé plus", clinique coopérative de Boundarou, infirmerie de la Société Béninoise de Brasserie, ONG CABM, et l'unité de santé de Banikanni.

L'échantillonnage était non aléatoire. La taille de l'échantillon a été calculée en utilisant la commande sskdlg (Reichenheim, 2000) du logiciel statistique STATA $^{\circledR}$. Ce calcul a été fait sur la base d'un kappa prévu de 0,65 , une prévalence du syndrome métabolique de $23 \%$ pour chaque groupe de critères, une différence envisagée minimum de 0,11 entre le kappa et ses bornes supérieures ou inférieures de l'intervalle de confiance de $95 \%$. En utilisant ces critères, la taille minimale de l'échantillon exigée était de 264 participants.

Ont été inclus dans l'étude, les sujets hypertendus adultes (âge $>18$ ans) consultant dans les formations sanitaires susmentionnées pendant la période d'étude. Ont été non inclus, les femmes enceintes, les sujets présentant une pathologie entrainant une augmentation du périmètre abdominal (tumeur, ascite, hernie) ou souffrant de coagulopathie ou n'ayant pas donné leur consentement.

Les variables étudiées étaient : l'âge, le sexe, l'indice de masse corporelle, la durée moyenne de l'hypertension artérielle (HTA), les antécédents personnels de diabète sucré, la ménopause, les composantes du SM selon les critères de la NCEP et l'IDF (tour de taille, 
pression artérielle, triglycéridémie, cholestérolémie HDL, glycémie à jeun).

\section{Recueil des données}

Le recueil des données a été fait à l'aide d'un questionnaire écrit et administré et par la consultation du carnet médical, l'examen clinique et les résultats d'analyses biochimiques. La mesure de la pression artérielle a été faite selon les recommandations de Bates et al. (2001) en position assise. La mesure des autres paramètres, poids, taille et tour de taille, a été effectuée selon le protocole de l'OMS (WHO, 1989). L'HTA a été définie par l'existence d'une pression artérielle supérieure ou égale à $140 \mathrm{mmHg}$ pour la systolique et/ou supérieure ou égale à $90 \mathrm{mmHg}$ pour la diastolique (Chobanian et al., 2003), ou par la prise d'un traitement hypotenseur quel qu'il soit.

\section{Prélèvement du sang et dosage des paramètres biochimiques}

Les prélèvements sanguins ont été réalisés les matins par ponction veineuse superficielle au pli du coude chez des patients à jeun depuis 10 à 12 heures d'horloge dans des tubes contenant du fluorure de sodiumoxalate (pour le dosage de la glycémie) et dans des tubes secs (dosage des autres paramètres). Les échantillons de sang ainsi obtenus (4 ml par tube) ont été acheminés au Laboratoire de Biochimie du Centre Hospitalier Départemental du Borgou pour les différents dosages. La glycémie a été dosée par la méthode enzymatique en point final à la glucose oxydase peroxydase (Trinder, 1969) avec les réactifs du laboratoire Span Diagnostics, la triglycéridémie par la méthode enzymatique en point final à la glycérol phosphate oxydase (Solera, 2000) avec des kits du laboratoire BIOLABO et la cholestérolémie HDL par la méthode de précipitation à l'acide phosphotungstique en présence d'ions magnésiums (Warnick et al., 1979) avec les kits du laboratoire ILO diagnostic.

\section{Analyse statistique}

Les données ont été analysées à l'aide du logiciel SPSS 17.0. Les résultats ont été présentés sous forme de proportions et de moyennes avec leurs écarts-types. La comparaison des proportions a été faite par le test de chi carré, les moyennes par le test $\mathrm{t}$ de Student et l'analyse de la variance (ANOVA). La concordance entre les critères de définition du syndrome métabolique a été évaluée avec l'utilisation du test Kappa et interprétée selon les valeurs références de Brennan et Silman (1992). La différence était significative pour $\mathrm{p}$ $<0,05$.

\section{RESULTATS}

Une population de 270 sujets adultes hypertendus avait été recrutée pour les besoins de notre étude. Celle-ci se composait de 150 femmes et de 120 hommes. Les caractéristiques cliniques de la population sont résumées dans le Tableau 1. Ce tableau montre que la valeur moyenne du tour de taille (en $\mathrm{cm}$ ) des hommes était la même que celle des femmes $(p=0,642)$. Les pressions artérielles systolique et diastolique moyennes $(\mathrm{mmHg})$ pour les femmes et les hommes étaient statistiquement identiques: $\mathrm{p}=0,394$ et 0,446 respectivement. Le Tableau 2 présente les paramètres biochimiques de la population d'étude. Il ressort de ce tableau que les glycémies, les triglycéridémies et les cholestérolémies HDL en (mmol/L) étaient statistiquement identiques dans les deux sexes: $\quad \mathrm{p}=0,884,0,378$ et 0,334 respectivement.

Selon les critères de définition de la FID, $188(69,6 \%)$ participants avaient le SM alors que $176(65,2 \%)$ l'avaient selon les critères de définition de la NCEP. La 
prévalence du SM était significativement plus élevée chez les femmes $(\mathrm{p}<0,001)$ en utilisant chaque critère de définition. En effet, elle était de $86 \%$ chez les femmes contre $59 \%$ chez les hommes selon les critères de la FID et de $76 \%$ chez les femmes contre $52 \%$ chez les hommes selon les critères de la NCEP. Le Tableau 3 montre la prévalence du syndrome métabolique par tranches d'âge et par sexe selon les deux critères de définition.

La concordance entre les deux définitions était de 0,41 (intervalle de confiance à $95 \%$ : 0,30 à 0,53 ) pour la population générale, de 0,30 (intervalle de confiance à $95 \%$ : 0,12 à 0,48$)$ chez les femmes et de 0,38 (intervalle de confiance à 95\%: 0,22 à 0,55) chez les hommes. Cette concordance entre les deux définitions était moyenne (kappa $=0,41)$ pour l'ensemble de la population d'étude et chez les hommes (kappa $=0,41$ ) alors qu'elle était faible chez les femmes (kappa =0,38). Le Tableau 4 montre la concordance entre les deux critères de définition selon le sexe et par tranches d'âge.

Tableau 1: Caractéristiques cliniques de la population d'étude, Parakou, 2010.

\begin{tabular}{|c|c|c|c|c|}
\hline Variables & $\begin{array}{l}\text { Femmes } \\
(n=150)\end{array}$ & $\begin{array}{l}\text { Hommes } \\
(n=120)\end{array}$ & $\mathbf{p}$ & $\begin{array}{c}\text { Total } \\
(n=270)\end{array}$ \\
\hline Moyenne d'âge en année $(\mathrm{m} \pm \mathrm{ET})^{\mathrm{a}}$ & $52,7 \pm 11,6$ & $53,6 \pm 10,9$ & 0,719 & $53,1 \pm 11,3$ \\
\hline $\mathrm{IMC}^{\mathrm{b}}(\mathrm{m} \pm \mathrm{ET})$ & $30,2 \pm 6,9$ & $26,8 \pm 4,7$ & $<0,001$ & $28,7 \pm 6,3$ \\
\hline Tour de taille en $\mathrm{cm}(\mathrm{m} \pm \mathrm{ET})$ & $95,1 \pm 14,5$ & $94,4 \pm 11,8$ & 0,642 & $94,8 \pm 13,3$ \\
\hline Ancienneté de l'HTA (année) ${ }^{\mathrm{c}}$ & $3(1-7)$ & $3(1-5,5)$ & 0,795 & $3(1-6)$ \\
\hline $\mathrm{PAS}^{\mathrm{d}}$ en $\mathrm{mmHg}( \pm \mathrm{ET})$ & $162,6 \pm 25,8$ & $160,2 \pm 19,7$ & 0,394 & $161,5 \pm 23,3$ \\
\hline $\mathrm{PAD}^{\mathrm{e}}$ en $\mathrm{mmHg}(\mathrm{m} \pm \mathrm{ET})$ & $97,6 \pm 16,3$ & $99 \pm 13,1$ & 0,446 & $98,22 \pm 14,9$ \\
\hline $\operatorname{ATCD}^{\mathrm{f}}$ de diabète $(\mathrm{n}, \%)$ & $10(6,7)$ & $17(14,2)$ & 0,041 & $27(10)$ \\
\hline Menopause $\left(\mathrm{n}^{\mathrm{g}}, \%\right)$ & $98(65,3)$ & $\mathrm{N} / \mathrm{A}^{\mathrm{h}}$ & $\mathrm{N} / \mathrm{A}$ & $98(36,3)$ \\
\hline
\end{tabular}

Tableau 2 : Paramètres biochimiques de la population d'étude, Parakou, 2010.

\begin{tabular}{ccccc}
\hline & $\begin{array}{c}\text { Femmes } \\
\left(\mathbf{n}^{\mathbf{b}}=\mathbf{1 5 0}\right)\end{array}$ & $\begin{array}{c}\text { Hommes } \\
(\mathbf{n}=\mathbf{1 2 0})\end{array}$ & $\mathbf{p}$ & $\begin{array}{c}\text { Total } \\
(\mathbf{n}=\mathbf{2 7 0})\end{array}$ \\
\hline $\begin{array}{c}\text { Glycémie } \\
(\mathrm{m} \pm \mathrm{ET})^{\mathrm{a}}(\mathrm{mmol} / \mathrm{L})\end{array}$ & $5,84 \pm 3,45$ & $5,78 \pm 2,72$ & 0,884 & $5,78 \pm 3,17$ \\
\hline $\begin{array}{c}\text { Triglycéridémie } \\
(\mathrm{m} \pm \mathrm{ET})(\mathrm{mmol} / \mathrm{L})\end{array}$ & $1,42 \pm 0,83$ & $1,33 \pm 0,84$ & 0,378 & $1,38 \pm 0,83$ \\
\hline $\begin{array}{c}\text { Cholestérolémie HDL } \\
(\mathrm{m} \pm \mathrm{ET})(\mathrm{mmol} / \mathrm{L})\end{array}$ & $0,80 \pm 0,36$ & $0,88 \pm 0,52$ & 0,334 & $0,83 \pm 0,44$ \\
\hline${ }^{\mathrm{a}}$ Moyenne \pm écart-type ; ${ }^{b}$ Effectif & & &
\end{tabular}


M. GOMINA ASSOUMANOU et al. /Int. J. Biol. Chem. Sci. 6(4): 1419-1427, 2012

Tableau 3: Prévalence du syndrome métabolique par tranches d'âge et par sexe selon les critères de la FID et de la NCEP, Parakou, 2010.

\begin{tabular}{|c|c|c|c|c|c|c|c|c|}
\hline \multirow[b]{2}{*}{ Groupes d'âge } & \multicolumn{4}{|c|}{ Critères FID } & \multicolumn{4}{|c|}{ Critères NCEP } \\
\hline & $\begin{array}{c}\text { Femmes } \\
\mathbf{n}^{\mathrm{a}}(\%)\end{array}$ & $\begin{array}{c}\text { Hommes } \\
\text { n }(\%)\end{array}$ & $\mathbf{p}$ & $\begin{array}{l}\text { Total } \\
\text { n }(\%)\end{array}$ & $\begin{array}{c}\text { Femmes } \\
\text { n }(\%)\end{array}$ & $\begin{array}{c}\text { Hommes } \\
\text { n }(\%)\end{array}$ & $\mathbf{p}$ & $\begin{array}{l}\text { Total } \\
\text { n }(\%)\end{array}$ \\
\hline$<45$ ans & $31(89)$ & $8(35)$ & $<0,001$ & $39(67)$ & $26(74)$ & $9(39)$ & 0,013 & $35(60)$ \\
\hline $55-64$ ans & $32(91)$ & $16(46)$ & $<0,001$ & 48 (69) & $28(80)$ & $19(54)$ & 0,041 & 47 (67) \\
\hline$>65$ ans & $21(70)$ & $15(75)$ & 0,758 & $36(72)$ & $18(60)$ & $12(60)$ & 1,00 & $30(60)$ \\
\hline
\end{tabular}

Tableau 4 : Concordance entre les critères de la FID et de la NCEP selon le sexe et par tranches d'âge, Parakou, 2010.

\begin{tabular}{lccc}
\hline Groupe d'âge & Femmes & Hommes & Total \\
\hline$<45$ ans & $0,54(0,21 \text { à } 0,87)^{\text {a }}$ & $0,53(0,18$ à 0,89$)$ & $0,63(0,42$ à 0,83$)$ \\
$45-54$ ans & $0,04(-0,26$ à 0,33$)$ & $0,43(0,16$ à 0,70$)$ & $0,40(0,20$ à 0,61$)$ \\
$55-64$ ans & $0,32(-0,08$ à 0,71$)$ & $0,15(-0,17$ à 0,47$)$ & $0,31(0,08$ à 0,55$)$ \\
$>\quad 65$ ans & $0,20(-0,15$ à 0,55$)$ & $0,44(0,05$ à 0,84$)$ & $0,30(0,3$ à 0,56$)$ \\
Total & $0,30(0,12$ à 0,48$)$ & $0,38(0,22$ à 0,55$)$ & $0,41(0,30$ à 0,53$)$ \\
\hline \multicolumn{2}{c}{${ }^{a}$ Intervalle de confiance } & &
\end{tabular}




\section{DISCUSSION}

Nous avons réalisé une étude transversale à visée descriptive avec recueil prospectif des données dans les formations sanitaires de la ville de Parakou en République du Bénin, qui nous a permis de déterminer la prévalence du SM chez les hypertendus adultes selon les critères de la Fédération Internationale du Diabète (FID) et de la National Cholesterol Education Programme (NCEP) et d'évaluer la concordance entre ces deux critères. La prévalence du syndrome métabolique était élevée en utilisant les deux critères diagnostiques mais l'était plus avec les critères de la FID. Ces taux étaient statistiquement plus élevés chez les femmes que chez les hommes, indépendamment des critères utilisés. La concordance entre les deux définitions était moyenne pour l'ensemble de la population d'étude et chez les hommes alors qu'elle était faible chez les femmes.

Notre étude a quelques limites à prendre en compte dans l'interprétation de nos résultats. En effet, les valeurs seuils pour le tour de taille appliquées dans notre population d'étude étaient celles des caucasiens. Celles-ci pourraient ne pas être appropriées pour caractériser l'obésité abdominale dans notre population, étant donné les différences probables de phénotype entre les caucasiens et les africains mélanodermes.

Dans notre population d'étude, la valeur moyenne du tour de taille des hommes était la même que celle des femmes ( $p=$ 0,642 ). Ce résultat est semblable à celui rapporté par Kelliny et al. (2003) aux Seychelles. A l'inverse, il est différent de celui trouvé par Akintunde et al. (2011) au Nigéria où, la moyenne du tour de taille était plus élevée chez les femmes, 103,7 \pm 11,5 vs $93,4 \pm 12,4 \quad(p=0,013)$. Les pressions artérielles systolique et diastolique moyennes étaient statistiquement identiques dans les deux sexes dans notre étude $(\mathrm{p}=0,394)$. Ce résultat est semblable à ceux rapportés par Akintunde et al. (2011) au Nigéria et Kelliny et al. (2003) aux Seychelles.

Dans notre étude, les glycémies, les triglycéridémies et les cholestérolémies HDL étaient statistiquement identiques dans les deux sexes. Ce résultat est similaire à celui de Akintunde et al. (2011) au Nigéria qui est de $5,3 \pm 1,6 \mathrm{mmol} / \mathrm{L}$ pour les femmes vs $5,7 \pm$ $2,3 \mathrm{mmol} / \mathrm{L}$ pour les hommes $(\mathrm{p}=0,105)$ pour les glycémies, $1,2 \pm 0,5 \mathrm{mmol} / \mathrm{L}$ pour les femmes vs 1,2 $\pm 0,4 \mathrm{mmol} / \mathrm{L}$ pour les hommes $(\mathrm{p}=0,648)$ pour les triglycéridémies et 1,2 $\pm 0,48$ pour les femmes vs 1,2 $\pm 0,43$ pour les hommes $(\mathrm{p}=0,38)$ pour les cholestérolémies HDL. Des résultats similaires ont été rapportés par Kelliny et al. (2003) aux Seychelles. Ces observations peuvent s'expliquer par la distribution raciale des paramètres lipidiques. En effet, au sein d'une même race, les valeurs des substrats lipidiques varient peu alors que cette variation est significative d'une race à une autre (Després et al., 2000).

Nous n'avons connaissance d'aucun autre taux rapporté de la prévalence du SM parmi les patients hypertendus béninois. Nous avons noté une prévalence élevée du SM dans notre population d'étude. Utilisant les critères de la FID, nous avons eu une prévalence de $69,6 \%$. Ce résultat est inférieur aux $82,4 \%$ rapportés par Bulhões et Araújo (2007) au Brésil et aux 81,2\% retrouvés par PaterninaCaicedo et al. (2009) en Colombie utilisant la même définition. Cette différence pourrait être due au fait qu'aussi bien au Brésil qu'en Colombie, les patients hypertendus avaient tous une obésité abdominale. A l'inverse, cette prévalence est supérieure aux 42,5\% rapportés par Akintunde et al. (2011) au Nigéria. Avec la définition de la NCEP nous avons eu une prévalence de $65,2 \%$. Ce résultat est similaire aux $71,6 \%$ observés au Brésil 
par Bulhões et Araújo (2007). Mais en Chine Chih-Neng et al. (2005) rapportent une prévalence de $47,9 \%$ selon les critères de la NCEP, inférieure aux $65,2 \%$ obtenues dans notre étude. Ceci pourrait être expliqué par les valeurs basses du tour de taille considérées pour définir l'obésité en Chine (Chih-Neng et al., 2005). Cette prévalence est supérieure aux $33,5 \%$ et $35 \%$ rapportés respectivement par Okpechi et al. (2007) et Akintunde et al. (2011) au Nigéria. Nos résultats, aussi bien que ceux des études chinoise, nigériane et brésilienne, relèvent que la prévalence du SM chez les patients hypertendus semble être indépendante de la race pendant que la prévalence reste très élevée indépendamment des critères de définition utilisés. En accord avec les études antérieures réalisées par Ford et al. (2002) et Ford (2005) dans la population générale, la présente étude a trouvé que selon les deux définitions, la prévalence du SM augmentait avec l'âge dans une population hypertendue. Cette observation peut être attribuée à une tendance à une augmentation relative avec l'âge de chacun des composants $\mathrm{du}$ syndrome métabolique. En outre, l'augmentation relative avec l'âge de la résistance à l'insuline (facteur prédisposant) a été montrée par plusieurs auteurs (Lorenzo et al., 2003). Dans notre étude, la prévalence du syndrome métabolique selon les critères de définition de la NCEP $(65,2 \%)$ était inférieure à celle selon les critères de la FID $(69,6 \%)$. Ce résultat était surtout dû au seuil de tour de taille différent entre les deux définitions. En effet, la valeur seuil du tour de taille, critère obligatoire pour la définition de la FID (> 94 $\mathrm{cm}$ pour les hommes, > $80 \mathrm{~cm}$ pour les femmes) est basse comparée à celle de la NCEP (>102 cm pour les hommes, > $88 \mathrm{~cm}$ pour les femmes). Ce constat est compatible à ceux faits par Bulhões et Araújo (2007) et Akintunde et al. (2011) dans lesquels la prévalence du SM est plus élevée selon la définition de la FID que celle de la NCEP. Nous avons observé une prédominance du SM chez les femmes; ce résultat est similaire à l'observation de Chih-Neng et al. (2005) en Chine. La prévalence élevée du SM chez les femmes est probablement liée à l'obésité, les femmes étant plus obèses que les hommes dans notre étude ( $\mathrm{p}<0,001$ pour les IMC).

La concordance entre les deux définitions utilisées dans notre étude était moyenne selon les critères de classification de Brennan et Silman (1992). Ce résultat est différent de celui observé par Bulhões et Araújo (2007) qui ont rapporté une bonne concordance en utilisant les mêmes critères de classification et les mêmes définitions que nous. Ce constat pourrait être expliqué par le fait que la moyenne du tour de taille de notre population était en dessous de la valeur normale exigée pour la définition de la NCEP alors que la condition obligatoire pour la définition de la FID qui est l'obésité abdominale a été remplie.

La prévalence du syndrome métabolique dans la présente étude est élevée. Notre étude, contribue à l'établissement d'une base de données épidémiologiques du syndrome métabolique chez les hypertendus au Bénin et dans la région africaine. Nos résultats soulignent l'accroissement de la prévalence des maladies non transmissibles en région africaine compatible à l'augmentation de l'obésité, qui est en train de devenir un des principaux facteurs de risque pour les maladies cardiovasculaires en région africaine (Ikem et Sumpo, 2011).

\section{RÉFÉRENCES}

Akintunde AA, Olugbenga EA, Akinwusi OP, Opadijo GO. 2011. Metabolic syndrom: comparison of occurrence using three definitions in hypertensive patients. $C M \& R, 9(1): 26-31$. 
Athyros VG, Bouloukos VI, Pehlivanidis AN. 2005. The prevalence of the metabolic syndrome in Greece: The MetS-Greece Multicentre Study. Diabetes, Obesity and Metabolism, 7: 397-405.

Bates B, Bickley S, Hoekelman A. 2001. Guide de l'Examen Clinique. Arnette: Paris.

Bulhões K, Araújo L. 2007. Metabolic syndrome in hypertensive patients: correlation between anthropometric data and laboratory findings. Diabetes Care, 30: 1624-1626.

Brennan P, Silman A. 1992. Statistical methods for assessing observer variability in clinical measures. BMJ, 304: 14911494.

Chih-Neng H, Ya-Chun C, Tzung-Dau W. 2005. Prevalence and Characterstics of the Metabolic Syndrome in chinese hypertensive patients: A Hospital-Based Observation. Acta Cardiol. Sin., 21: 8997.

Chobanian AV, Bakris GL, Black HR. 2003. Seventh report of the Joint National Committee on prevention, detection, evaluation and treatment of high blood pressure. J. Hypertens., 42: 1206-1252.

Després JP, Couillard C, Gagnon J, Bergeron J, Arthur S, James S. 2000. Race, visceral adipose tissue, plasma lipids, and lipoprotein lipase activity in men and women. the health, risk factors, exercise training, and genetics (HERITAGE) family study. Arterioscler. Thromb. Vasc. Biol., 8: 2-7.

Fezeu L, Balkau B, Kengne AP, Sobngwi E, Mbanya JC. 2007. Metabolic syndrome in a sub Saharan African setting; central obesity may be the key determinant. Atherosclerosis, 193: 70-76.

Ford ES, Giles WH, Dietz WH. 2002. Prevalence of the metabolic syndrome among US adults: findings from the Third
National Health and Nutrition Examination Survey. JAMA, 287: 356359.

Ford ES. 2005. Prevalence of the metabolic syndrome defined by the International Diabetes Federation among adults in the US. Diabetes Care, 28(11): 2745-2749.

Grundy SM, Brewer BH, Cleeman IJ, Smith CS, Lenfant C. 2004. Definition of Metabolic Syndrome: Report of the National Heart, Lung, and Blood Institute/American Heart Association Conference on Scientific Issues Related to Definition. Circulation, 109: 433-438.

Hydrie MZ, Shera AS, Fawwad A. 2009. Prevalence of the metabolic syndrome in urban Pakistan (Karachi): comparison of newly. Syndr. Relat. Disord., 7: 119-124.

Ikem I, Sumpo BE. 2011. Cardiovascular disease: the new epidemic in sub-Saharan Africa. Vascular, 19(6): 301-307.

Kannel WB. 2000. Risk stratification in hypertension: new insights from the Framingham Study. Am. J. Hypertens., 13: $3 \mathrm{~S}-10 \mathrm{~S}$.

Kelliny C, William J, Riesen W, Paccaud F, Bovet P. 2008. Metabolic syndrome according to different definitions in a rapidly developing country of the African region. Cardiovascular Diabetology, 7: 27.

Lorenzo C, Okoloise M, Williams K, Stern MP, Haffner SM. 2003. The Metabolic Syndrome as Predictor of Type 2 Diabetes: the San Antonio heart study. Diabetes Care, 26(11): 3153-3159.

Okpechi IG, Pascoe MD, Swanepoel CR, Rayner BL. 2007. Microalbuminuria and the metabolic syndrome in nondiabetic black Africans. Diab. Vasc. Dis. Res., 4: 365-367.

Paternina-Caicedo AJ, Alcalá-Cerra G, Paillier-Gonzales J, Romero-Zarante AM, Alvis-Guzmán N. 2009. Agreement 
between three definitions of metabolic syndrome in hypertensive patients. Rev. Salud. Publica (Bogota), 11(6): 898-908.

Pollex RL, Hanley AJ, Zinman B. 2006. Metabolic syndrome in aboriginal Canadians: Prevalence and genetic associations. Atherosclerosis, 184: 121129.

Reichenheim ME. 2000. Sample size for the kappa-statistic of interrater agreement. Stata Technical Bulletin, 58: 41-45.

Solera M-L. 2000. Métabolisme des lipides et des lipoprotéines. In Biochimie Clinique, Valdiguié P (ed). Editions Médicales Internationales : Cachan; 160-186.
Trinder P. 1969. Determination of blood glucose using an oxidase-peroxidase system with a non-carcinogenic chromogen. J. Clin. Path., 22: 158-161.

Warnick GR, Cheung MC, Albers JJ. 1979. Comparison of current methods for highdensity lipoprotein cholesterol quantification. Clin. Chem., 25(4): 596604.

World Health Organisation. 1989. Measuring Obesity: Classification and Distribution of Anthropometric Data. WHO: Copenhagen. 